
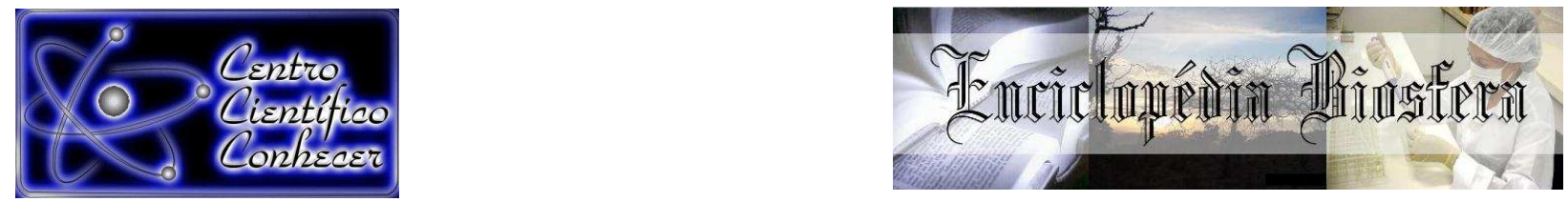

\title{
INFLUÊNCIA DA ASSISTÊNCIA PRÉ-NATAL SOBRE O CONSUMO ALIMENTAR, GANHO DE PESO MATERNO E DESFECHOS GESTACIONAIS
}

\author{
Liliane Gehring $^{1}$; Karen Suelen Fernandes Modafares ${ }^{2}$; Andréa Grano Marques ${ }^{3}$ \\ ${ }^{1}$ Pós-graduanda do Programa de Mestrado em Promoção da Saúde do Centro \\ Universitário de Maringá - UniCesumar, Maringá, Paraná, Brasil. \\ ${ }^{2}$ Graduanda do Curso de Nutrição da Faculdade Integrado, Campo Mourão, \\ Paraná, Brasil. \\ ${ }^{3}$ Profa Doutora do Programa de Mestrado em Promoção da Saúde do Centro \\ Universitário de Maringá - UniCesumar, Maringá, Paraná, Brasil. \\ (andreagrano298@hotmail.com)
}

Recebido em: 08/09/2015 - Aprovado em: 14/11/2015 - Publicado em: 01/12/2015 DOI: http://dx.doi.org/10.18677/Enciclopedia_Biosfera_2015_043

\begin{abstract}
RESUMO
A assistência pré-natal consiste em um importante acompanhamento que visa prevenir a ocorrência de desfechos perinatais indesejáveis. O objetivo desta pesquisa foi avaliar o consumo alimentar, o ganho de peso materno e os desfechos gestacionais, via de parto e peso ao nascer do concepto, em gestantes que realizaram pré-natal. A amostra foi composta por 105 gestantes internadas para a realização do parto em um Hospital Maternidade no Centro-Oeste do Paraná. A coleta dos dados maternos e do concepto ocorreu no puerpério imediato (até 48 horas pós-parto). Foi realizada avaliação do estado nutricional, por meio das medidas antropométricas e da avaliação do consumo alimentar. Os resultados foram agrupados em duas categorias, de acordo com a faixa etária. O ganho médio de peso médio foi de $14,7 \mathrm{~kg}$, a maior inadequação de ganho de peso ocorreu entre as gestantes que iniciaram a gestação em sobrepeso e obesidade. A ingestão calórica média foi de $2.275 \mathrm{kcal} /$ dia e houve excesso no consumo de carnes e derivados. Em relação aos desfechos a taxa de partos cirúrgicos foi $72,4 \%$ e 0 crescimento fetal foi adequado em $71,4 \%$ dos conceptos. Verificou-se que a assistência pré-natal foi efetiva para a prevenção de intercorrências gestacionais, entretanto não foi efetiva para os desfechos gestacionais. A taxa de parto cirúrgico foi extremamente elevada, o ganho de peso materno foi acima do recomendado em gestantes com sobrepeso e obesidade e o percentual de recém-nascidos pequenos para a idade gestacional foi acima do esperado, principalmente no grupo de puérperas adultas.
\end{abstract}

PALAVRAS-CHAVE: assistência pré-natal; estado nutricional; hábito alimentar 


\title{
NUTRITION IN PREGNANCY: EVALUATION OF GESTATIONAL WEIGHT GAIN AND RELATIONSHIP WITH COMPLICATIONS IN PREGNANCY AND CHILDBIRTH
}

\begin{abstract}
The prenatal care is an important pregnancy assistance that prevents the undesirable perinatal mortality. This research work evaluates the dietary habits, the weight gain of pregnant mothers and the end of the pregnancy of women followed in the prenatal program. The sample comprised of 105 inmate pregnants from a maternity hospital of the Midwest of Paraná State (Brazil). Data of mothers and their child were taken immediately after the child-birth. The nutritional evaluation considered the anthropometric weights and evaluated the dietary habits. The results were separated in two groups according to mother ages. The average weight gain of these mothers was $14.7 \mathrm{Kg}$. Most of the mothers with overweight or obesity had an inadequate gain of weight. The caloric average was $2,275 \mathrm{kcal} /$ day exceeding in meat and derivatives. $72.4 \%$ were chirurgic child-births and $74.1 \%$ of the newborns presented appropriate fetal development. According to the present research study, the prenatal assistance was effective to prevent pregnancy complications, but not on the conclusion of the pregnancy. The chirurgic child-births rates were significant high, the weight gain of overweight or obese mothers was above recommended, and the rate of small newborns was above of expected, specially in group of adult women.
\end{abstract}

KEYWORDS: prenatal assistance; nutritional status; dietary habits

\section{INTRODUÇÃO}

O acompanhamento do estado nutricional da gestante, por meio das medidas antropométricas e da avaliação do consumo alimentar, tem sido recomendado em função da crescente prevalência de intercorrências associadas ao ganho de peso gestacional excessivo ou insuficiente, assim como pela relação existente entre o índice de massa corporal e os desfechos gestacionais (NELSON et al., 2010; BLOMBERG, 2011).

A obesidade materna é considerada fator de risco relacionado às complicações que podem ocorrer no período gestacional, como diabetes gestacional, síndromes hipertensivas da gravidez, trabalho de parto prolongado, desproporção céfalo-pélvica e indicação de parto cirúrgico (NELSON et. al, 2010; DODD et al., 2010; YAZDANI et al., 2012), podendo acarretar para o recém-nascido macrossomia e sofrimento fetal (GONÇALVES et al., 2012).

O ganho de peso na gestação abaixo do recomendado pode resultar, para o concepto, em crescimento intrauterino restrito (NOMURA et al., 2012). O peso ao nascer é um importante indicador do estado de saúde do recém-nascido, estando associado a complicações clínicas no período neonatal, como doenças infecciosas, respiratórias e atraso do crescimento (NASCIMENTO et al., 2012), assim como à mortalidade nos primeiros anos de vida (PADILHA et al., 2007).

A gravidez ocorre ao longo da vida reprodutiva da mulher, entretanto em idade precoce há um aumento do risco de nascimento de bebês com baixo peso, resultado do crescimento intrauterino restrito (SURITA et al., 2011). A vulnerabilidade em termos nutricionais ocorre em função do aumento do requerimento de nutrientes, tanto para o crescimento e desenvolvimento da adolescente quanto para atender às necessidades nutricionais do feto em formação. A maneira mais eficaz de avaliar os fatores que levam a um ganho de peso 
inadequado é a investigação dos hábitos alimentares, avaliando-se os aspectos quantitativos e qualitativos da dieta (GUIMARÃES \& GALANTE, 2009).

Os desfechos gestacionais são resultantes de uma complexa rede de determinantes biológicos, socioeconômicos e assistenciais. Dentre os biológicos destaca-se o estado nutricional materno, cujo acompanhamento, por meio da avaliação do consumo alimentar e do monitoramento do ganho de peso, diminui os riscos adicionais à gestação contribuindo para desfechos favoráveis para a mãe e o concepto. O Ministério da Saúde preocupado com a saúde da mulher e da criança no período gestacional implementou um novo modelo de assistência, a Rede Cegonha, que visa garantir o acolhimento e a classificação de risco e de vulnerabilidade, ampliando o acesso e a qualidade do pré-natal assegurando às mulheres atenção humanizada à gravidez, ao parto e ao puerpério e às crianças o direito ao nascimento seguro e ao crescimento e desenvolvimento saudáveis (BRASIL, 2011).

Assim, a assistência pré-natal contribui para desfechos gestacionais mais favoráveis à saúde materna e infantil controlando os fatores de risco que resultam em complicações, tanto para a mulher quanto para o bebê. O Institute of Medicine (2009) reconhece o peso pré-gestacional como um dos principais determinantes na recomendação do ganho de peso durante a gestação, baseada no índice de massa corporal (IMC). Portanto, como o pré-natal preconiza o desenvolvimento de ações educativas, realizadas nas Unidades Básicas de Saúde, em grupo ou individualmente, deve proporcionar às gestantes orientações sobre ganho de peso, assim como os tipos de parto, vantagens, desvantagens e riscos (VIEIRA et al., 2011) e os cuidados com o bebê (SOUZA et al. 2011).

O objetivo desta pesquisa foi avaliar o consumo alimentar, o ganho de peso materno e os desfechos gestacionais, via de parto e peso ao nascer do concepto, em gestantes que realizaram pré-natal.

\section{MATERIAL E MÉTODOS}

A pesquisa foi avaliada e aprovada pelo Comitê de Ética em Pesquisa da Faculdade Integrado de Campo Mourão, sob o parecer de número 327.323/2013. Foram incluídas na amostra 105 gestantes internadas para a realização do parto em um Hospital Maternidade no Centro-Oeste do Paraná, que concordaram em participar do estudo e assinaram o Termo de Consentimento Livre e Esclarecido (TCLE). Utilizou-se como critério de exclusão internamentos nos finais de semana, dados incompletos nos prontuários médicos e nas fichas de acompanhamento do pré-natal. O estudo foi realizado no período de junho a agosto de 2013.

A coleta dos dados ocorreu no puerpério imediato (até 48 horas pós-parto), sendo utilizada a anamnese hospitalar validada por GUIMARÃES \& GALANTE (2009). Os dados maternos referentes à via de parto, intercorrências na gestação, complicações no parto e peso corporal da gestante ao final da gestação foram obtidos a partir do prontuário médico do Hospital Maternidade.

A ficha de acompanhamento do pré-natal forneceu o histórico do ganho de peso materno, assim como os dados de altura e peso pré-gestacional que foram utilizados para o cálculo do índice de massa corporal (IMC) pré-gestacional. Foi adotada a recomendação do ganho de peso materno proposta pelo Institute of Medicine (2009) baseada no índice de massa corporal (IMC) pré-gestacional: IMC inferior a $18,5 \mathrm{~kg} / \mathrm{m}^{2}$ ganho de peso entre 12,5 a 18,0kg; IMC de 18,5 a $24,9 \mathrm{~kg} / \mathrm{m}^{2}$ ganho de peso entre 11,5 a $16,0 \mathrm{~kg}$; IMC de 25,0 a $29,9 \mathrm{~kg} / \mathrm{m}^{2}$ ganho de peso entre 7,0 a 11,5kg; IMC acima de $305 \mathrm{~kg} / \mathrm{m}^{2}$ ganho de peso entre 5,0 a 9,0kg. O ganho de 
peso gestacional foi calculado utilizando-se o peso corporal da gestante ao final da gestação menos o peso pré-gestacional, sendo posteriormente classificado como insuficiente, adequado ou em excesso, de acordo com o recomendado.

Para a avaliação do consumo alimentar foi realizada entrevista com as puérperas, sendo aplicados os seguintes instrumentos: registro alimentar habitual e questionário de frequência alimentar referente ao terceiro trimestre da gestação. Os dados qualitativos e quantitativos coletados foram convertidos em porções diárias e avaliados segundo a recomendação da pirâmide alimentar adaptada para a população brasileira (BRASIL, 2008). Para determinação do consumo calórico, as porções consumidas diariamente foram multiplicadas pelo valor calórico de cada grupo de alimentos da pirâmide alimentar e comparadas com as necessidades individuais estimadas para gestantes, de 2.855 calorias para o terceiro trimestre, de acordo com as recomendações de RASMUSSEN et al. (2009).

Foram considerados como desfechos gestacionais a via de parto e o peso ao nascer do concepto. As taxas de parto cirúrgico foram analisadas tendo como referência os valores preconizados pela WORLD HEALTH ORGANIZATION (1985). Os dados clínicos do recém-nascido (idade gestacional, peso e comprimento) foram coletados na ficha de declaração de nascidos vivos e classificados segundo as curvas de crescimento fetal de LUBCHENCO et al. (1970), com os seguintes pontos de corte: Pequeno para a Idade Gestacional (PIG) < percentil 10, Adequado para a Idade Gestacional (AIG) entre os percentis 10 a 90, Grande para a Idade Gestacional (GIG) > percentil 90 .

As puérperas foram divididas em dois grupos de acordo com a faixa etária, grupo um composto por 26 gestantes adolescentes entre 14 e 19 anos; e grupo dois por 79 gestantes adultas entre 20 e 35 anos. Os dados foram tabulados e analisados por meio do aporte estatístico do Microsoft Excel 2010. Utilizou-se porcentagem (\%), média e desvio padrão para a apresentação dos resultados.

\section{RESULTADOS E DISCUSSÕES}

A média de idade das puérperas adolescentes que compuseram a amostra deste estudo foi de 17 anos e das adultas 26 anos, sendo 24 anos a média geral. A classificação do índice de massa corporal pré-gestacional em 59\% das gestantes foi eutrofia, desnutrição em 13\%, sobrepeso em 18\% e obesidade em 9,5\%. PADILHA et al. (2007) relataram resultados semelhantes, pois $68,2 \%$ das gestantes avaliadas não apresentaram desvio ponderal pré-gestacional, 6,2\% baixo peso, 19,9\% sobrepeso e a menor porcentagem também foi composta por mulheres obesas, $5,5 \%$.

A associação entre a obesidade pré-gestacional e os hábitos alimentares inadequados foi relatada por ANDRETO et al., (2006) como fator de risco à gestação, tendo consequências a curto e a longo prazo para a mãe e o bebê. $O$ sobrepeso antes da gestação pode aumentar em 54\% o risco de diagnóstico de hipertensão e pode dobrar o risco de acometimento por diabetes mellitus (CORREIA et al., 2011). Enquanto que o baixo peso pré-gestacional, associado ao ganho de peso insuficiente durante a gestação, resulta em neonatos pequenos para a idade gestacional (NOMURA et al., 2012).

A média de ganho de peso durante a gestação das mulheres avaliadas foi de $14,1 \mathrm{~kg}( \pm 4,8)$, entre as adolescentes o ganho de peso médio foi de $14,7 \mathrm{~kg}( \pm 4,7)$ e entre as adultas o ganho médio foi de $13,9 \mathrm{~kg}( \pm 4,7)$. Entre as gestantes avaliadas $25,8 \%$ tiveram ganho de peso insuficiente, $37,1 \%$ das gestantes apresentaram 
ganho de peso adequado e $37,1 \%$ das gestantes excederam no ganho de peso independente do estado nutricional.

MARANO et al. (2012) avaliaram os fatores determinantes do ganho de peso gestacional. Por um lado o ganho excessivo foi atribuído à hipertensão arterial e ao sobrepeso ou obesidade pré-gestacionais, por outro lado o ganho de peso abaixo do recomendado não foi associado às morbidades maternas e aos fatores sociodemográficos, comportamentais e reprodutivos.

O aumento de peso durante a gestação predispõe ao aumento de obesidade em mulheres, pois este peso extra mantém-se após a gestação (AMORIM et al., 2007). Os programas de assistência pré-natal devem priorizar o controle de ganho de peso na gestação, VITOLO et al. (2011) descreveram que a orientação nutricional influencia positivamente na diminuição do ganho de peso e da prevalência de obesidade. A Figura 1 apresenta o ganho médio de peso gestacional, recomendações mínimas e máximas, de acordo com o estado nutricional pré-gestacional materno.

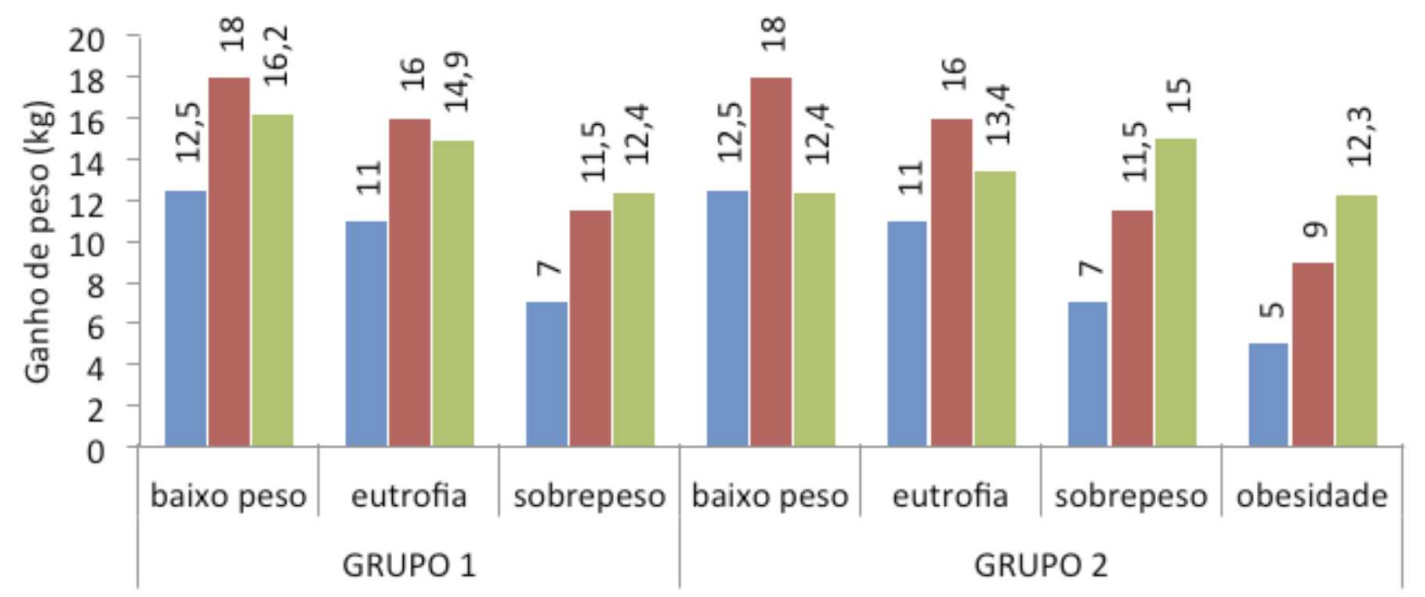

ganho de peso minimo recomendado ganho de peso maximo recomendado
ganho de peso encontrado

FIGURA 1- Ganho de peso gestacional, recomendações mínimas e máximas, de acordo com o estado nutricional pré-gestacional dos grupos avaliados em um Hospital Maternidade

A figura 1 demonstra o ganho de peso adequado entre as mulheres que iniciaram a gestação apresentando baixo peso e eutróficas, em ambos os grupos; ganho de peso excessivo entre as adolescentes que iniciaram a gestação com sobrepeso; e, no grupo das puérperas adultas àquelas que iniciaram a gestação com sobrepeso ou obesidade também ganharam peso além das recomendações. $O$ maior ganho de peso entre mulheres que iniciaram a gestação com excesso de peso pode ser atribuído ao balanço energético positivo pré-gestacional que predispõe ao ganho de peso.

FAZIO et al. (2011) verificaram ganho de peso acima do recomendado e diferenças estatisticamente significantes foram descritas quando o grupo de gestantes com sobrepeso ou obesidade foram comparadas ao grupo com eutrofia ou baixo peso, estes resultados corroboram com os achados do presente estudo. Entretanto, SEABRA et al. (2011) relataram que gestantes com sobrepeso ou obesidade ganharam menos peso comparadas às gestantes eutróficas ou com baixo peso, sendo as diferenças também estatisticamente significantes. Porém, a 
média de ganho de peso foi superior à recomendação. Está descrito na literatura que a inadequação do estado nutricional materno aumenta os riscos de intercorrências gestacionais que influenciam negativamente no curso da gestação (PADILHA et al., 2007).

O pré-natal, realizado por uma equipe multiprofissional, preconiza ações de promoção da saúde, prevenindo transtornos alimentares e psicoafetivos, melhorando a qualidade da assistência materna e do recém-nascido, prevenindo agravos e diminuindo os riscos de morbimortalidade materna e fetal (FALCONE et al., 2005). Portanto, a assistência pré-natal precisa desenvolver ações de orientação e de educação nutricional voltadas ao controle de ganho de peso gestacional.

A gestação e o puerpério são períodos onde a mulher está sujeita às influências baseadas em saberes provenientes da cultura popular, familiar e biomédica, que determinam às escolhas alimentares das gestantes e puérperas (BAIÃO \& DESLANDES, 2006). A importância da avaliação do consumo dietético de gestantes consiste em subsidiar o desenvolvimento de planos de ações eficazes no controle da qualidade da alimentação neste período de vulnerabilidade. O consumo alimentar das puérperas que compuseram a amostra deste estudo pode ser observado na tabela 1.

TABELA 1 - Consumo segundo as porções dos grupos alimentares e calorias ingeridas de puérperas

\begin{tabular}{lccc}
\hline $\begin{array}{l}\text { Grupo de } \\
\text { Alimentos } \\
\text { (recomendações) }\end{array}$ & $\begin{array}{c}\text { GRUPO 1 } \\
\text { Porções }\end{array}$ & $\begin{array}{c}\text { GRUPO 2 } \\
\text { Porções }\end{array}$ & $\begin{array}{c}\text { Média } \\
\text { Porções }\end{array}$ \\
\hline Cerais (5-9) & 7,5 & 6,0 & 6,7 \\
\hline Frutas (3-5) & 3,0 & 2,5 & 2,7 \\
\hline Hortaliças (4-5) & 4,0 & 4,0 & 4,0 \\
\hline Leguminosas (1) & 2,0 & 1,5 & 1,7 \\
\hline Leite e derivados (3) & 1,5 & 1,5 & 1,5 \\
\hline Óleos e gorduras (1-2) & 1,5 & 1,5 & 2,5 \\
\hline Açucares e doces (1-2) & 2,5 & 2,0 & 3,0 \\
\hline Carnes e ovos (1-2) & 3,0 & 3,0 & $2.275,7$ \\
\hline Kcal total (2.855) & $2.411,8$ & $2.139,7$ & \\
\hline
\end{tabular}

Em ambos os grupos de puérperas o consumo foi acima dos valores recomendados para os alimentos do grupo das carnes e ovos e do grupo das leguminosas, as gestantes adolescentes apresentaram maior consumo de açúcares e doces. Em ambos os grupos de gestantes destacou-se a baixa ingestão de leite e derivados, e entre as gestantes adultas observou-se consumo diminuído de frutas. Segundo FAZIO et al. (2011) o conhecimento dos detalhes do consumo dietético aprimora a qualidade do atendimento e das orientações realizadas, ou seja é preciso conhecer os hábitos alimentares para propor intervenções adequadas.

Em relação ao consumo energético as adolescentes apresentaram maior consumo de calorias, porém o consumo calórico de ambos os grupos de puérperas encontrou-se dentro das recomendações. A adequação do consumo calórico de gestantes é descrita nos estudos de BUSS et al. (2009) e NOMURA et al. (2012), corroborando com os resultados apresentados na tabela 1.

As puérperas foram questionadas quanto ao recebimento de orientação nutricional durante o pré-natal, $28,5 \%$ declararam que não receberam nenhum tipo de orientação e entre aquelas que receberam apenas $6,6 \%$ foram orientadas por nutricionista. VITOLO et al. (2011) avaliaram o impacto das orientações quanto às escolhas e combinações dos alimentos sobre o ganho de peso entre gestantes e 
relataram efeitos positivos na diminuição da velocidade de ganho de peso de gestantes com excesso de peso.

Verifica-se a necessidade de implantação de programas de orientação nutricional, visando a promoção de hábitos alimentares saudáveis e adequados, assim como o controle do ganho de peso no período gestacional. MARTINS \& BENICIO (2011) identificaram que o aumento do consumo de gordura saturada e alimentos processados especificamente aumentam a retenção de peso pós-parto.

Diante dos dados expostos, percebe-se a necessidade da orientação nutricional desde a puericultura com o objetivo de promover mudanças no estilo de vida e no padrão alimentar adotado pela população brasileira, que prioriza o consumo de produtos ricos em açúcar refinado, gorduras saturadas, refrigerantes e alimentos processados (VITOLO et al., 2011). Os resultados da prevalência da via de partos realizados no Hospital Maternidade pesquisada estão expostos na tabela 2.

TABELA 2 - Prevalência da via de partos realizados no Hospital Maternidade.

\begin{tabular}{lccc}
\hline Variáveis & $\begin{array}{c}\text { GRUPO 1 } \\
\mathrm{n}(\%)\end{array}$ & $\begin{array}{c}\text { GRUPO 2 } \\
\mathrm{n}(\%)\end{array}$ & $\begin{array}{c}\text { Média } \\
\mathrm{n}(\%)\end{array}$ \\
\hline Parto cirúrgico & $23(88,4)$ & $53(67,1)$ & $76(72,4)$ \\
\hline Parto normal & $3(11,6)$ & $26(32,9)$ & $29(27,6)$ \\
\hline TOTAL & $26(100,0)$ & $79(100,0)$ & $105(100,0)$ \\
\hline
\end{tabular}

Fonte: Hospital Maternidade

Na tabela 2 pode-se observar que o parto cirúrgico foi realizado em $72,4 \%$ das mulheres que compuseram a amostra deste estudo, apresentando percentual superior entre as parturientes adolescentes (88,4\%). Estudo realizado por SANTOS et al. (2012) descreveram o parto vaginal como sendo a via mais frequente entre as puérperas adolescentes avaliadas. GRAVENA et al. (2013) relataram que a taxa de parto cesáreo aumentou com o decorrer da idade materna, corroborando com 0 estudo de SANTOS et al. (2012).

Estudos relataram melhor desempenho obstétrico entre as puérperas adolescentes quando comparadas às puérperas adultas, em relação a via de parto (HELENA et al., 2008; SANTOS et al., 2012; GRAVENA et al., 2013). Entretanto, no presente estudo o percentual de parto cirúrgico foi extremamente elevado, principalmente entre as gestantes adolescentes. A taxa de parto cirúrgico preconizada pela WORLD HEALTH ORGANIZATION (1985) para a população de baixo risco deve ser mantida entre 10 a $15 \%$, sendo considerada inaceitável taxa acima de $40 \%$, mesmo em maternidades voltadas ao atendimento de gestação de alto risco.

Estudo realizado por REIS et al. (2014) que avaliaram o tipo de parto relacionado ao risco gestacional, considerando as repercussões tanto maternas quanto neonatais imediatas em um hospital universitário, relataram que a taxa total de cesarianas em uma amostra de 1606 partos foi de 38,3\%; sendo que a taxa de cesariana no grupo de mulheres com gestação de alto risco, foi de $57,8 \%$. NOMURA et al. (2004) descreveram taxa de parto cirúrgico em 56,5\%, também em gestações de alto risco. Ambos os estudos apresentaram valores inferiores ao demonstrado na tabela 2, cuja população não apresentou risco gestacional. Entretanto, este fenômeno não tem ocorrido isoladamente no País.

RATTNER et al. (2012) estudaram a magnitude e a tendência da taxa de cesáreas no Brasil, os resultados demonstraram que a proporção deste tipo de parto está em ascensão, a taxa, em 1994, foi de $32 \%$ e, em 2010 , foi de $52 \%$. Os autores 
relataram que as taxas de partos cirúrgicos são altas e também estão em ascensão nas parturientes adolescentes, que caracterizam uma população cujo futuro obstétrico deveria ser preservado evitando cicatrizes cirúrgicas uterinas.

O parto cesáreo é um procedimento cirúrgico, indicado quando ocorrem complicações durante a gravidez ou parto que colocam em risco a mãe, o bebê ou ambos. A cesariana não é isenta de riscos, estando associada a maior morbimortalidade materna e infantil, quando comparada ao parto normal. Há uma tendência ascendente da prematuridade em crianças nascidas por cesárea e uma tendência de redução nas crianças nascidas de parto normal, sendo que a prematuridade nas crianças em 2010 foi de 7,8\% em partos cesáreos e 6,4\% em partos normais (RATTNER et al., 2012).

A média de peso encontrado entre os recém-nascidos neste estudo foi de 3.200 gramas $( \pm 400 \mathrm{~g})$. GUERRA et al. (2007) relataram ganho de peso de 3.105 gramas $( \pm 391,8 \mathrm{~g})$ em recém-nascidos de puérperas adolescentes, enquanto que SEABRA et al. (2011) relataram média de $3.291,3$ gramas $( \pm 455,2 \mathrm{~g})$ do peso ao nascer do concepto em puérperas adolescentes e adultas de baixo e médio risco. Portanto, a média de peso encontrada no presente estudo apresenta valor intermediário quando comparada aos valores dos dois estudos.

A tabela 3 apresenta o resultado da avaliação e classificação dos RNs em relação ao crescimento fetal.

TABELA 3 - Classificação de crescimento fetal de bebês nascidos em Hospital Maternidade.

\begin{tabular}{cccc}
\hline Variáveis & $\begin{array}{c}\text { GRUPO 1 } \\
\mathrm{n}(\%)\end{array}$ & GRUPO 2 & $\begin{array}{c}\text { Geral } \\
\mathrm{n}(\%)\end{array}$ \\
\hline PIG & $5(19,3)$ & $19(24,5)$ & $24(22,8)$ \\
\hline AIG & $19(73,1)$ & $56(70,0)$ & $75(71,4)$ \\
\hline GIG & $2(7,6)$ & $4(5,5)$ & $6(5,8)$ \\
\hline TOTAL & $26(100,0)$ & $79(100,0)$ & $105(100,0)$ \\
\hline
\end{tabular}

Fonte: Hospital Maternidade

O peso do recém-nascido é influenciado pelo ganho de peso materno, quanto maior o ganho de peso gestacional maior o risco de macrossomia, principalmente com ganho acima de $17 \mathrm{Kg}$ (GONÇALVES et al., 2012). A tabela 3 demonstra $5,8 \%$ de recém-nascidos considerados grandes para a idade gestacional, sendo maior 0 percentual no grupo de puérperas adolescentes (7,6\%). Este resultado possivelmente está relacionado com o ganho de peso materno durante a gestação, pois $37,1 \%$ das puérperas que compuseram a amostra deste estudo apresentaram ganho de peso excessivo, sendo maior a média do ganho de peso no grupo das puérperas adolescentes quando comparadas ao grupo das adultas, respectivamente, $14,7 \mathrm{~kg}( \pm 4,7)$ e $13,9 \mathrm{~kg}( \pm 4,7)$.

$\mathrm{Na}$ tabela 3 pode-se observar que o percentual de bebês pequenos para a idade gestacional foi $22,8 \%$, considerando-se ambos os grupos, puérperas adolescentes e adultas. NOMURA et al. (2012) descreveram o nascimento de bebês pequenos para a idade gestacional em $24,3 \%$ das puérperas cuja gestação foi considerada de alto risco.

O percentual de recém-nascidos pequenos para a idade gestacional, no grupo das puérperas adolescentes, apresentou valor inferior (19,3\%), quando comparado ao valor médio das puérperas adultas (24,5\%). HELENA et al. (2008) realizaram estudo que avaliou e comparou o peso ao nascer de conceptos de gestantes adolescentes e adultas, diferenças estatisticamente significantes foram 
descritas pelos autores. As adolescentes apresentaram o percentual de $19,9 \%$ de bebês com baixo peso ao nascer, valor que corrobora com o resultado deste estudo $(19,3 \%)$, entretanto os autores descreveram que as mulheres adultas apresentaram $14,2 \%$, valor bem abaixo do encontrado no presente estudo $(24,5 \%)$.

Embora o esperado fosse encontrar maior percentual de baixo peso ao nascer no grupo das puérperas adolescentes, está descrito na literatura que o efeito da gestação na adolescência sobre os desfechos gestacionais são controversos, pois as intercorrências gestacionais são moduladas pelo grau de vulnerabilidade ao qual a adolescente possa estar exposta (SAUNDERS et al., 2009). GUIMARÃES et al. (2013) investigaram a gravidez na adolescência como fator de risco para baixo peso ao nascer, considerando variáveis biológicas, sociais e assistenciais. Os autores relataram fator de risco para baixo peso ao nascer no grupo de mães adolescentes sem companheiro.

Considerando que nesta investigação as gestações eram de baixo risco e todas iniciaram precocemente o pré-natal realizando mais de seis consultas, 0 percentual de bebês pequenos para a idade gestacional $(22,8 \%)$ foi superior ao esperado. Estudos demonstraram que o pré-natal confere proteção à ocorrência de baixo peso ao nascer (SANTOS et al., 2008; SANTOS et al., 2012), entretanto, a qualidade das orientações recebidas durante o pré-natal precisa ser considerada. $O$ parto cesário também exerce importante influência no nascimento de bebês abaixo do peso, pois a intervenção cirúrgica interrompe a gestação (SANTOS et al., 2012). No presente estudo, a média da idade gestacional foi 38 semanas, independente da faixa etária materna, o que pode ter aumentado o número de recém-nascidos com baixo peso.

ANVERSA et al. (2012) analisaram a qualidade da assistência pré-natal em um município cujo sistema de saúde era composto por 31 unidades, 18 Unidades Básicas de Saúde Tradicionais e 13 que adotaram a Estratégia Saúde da Família, sendo considerado o início do pré-natal, o número de consultas, a realização de procedimentos conforme manual técnico do Ministério da Saúde e de exames laboratoriais entre $28^{a}$ e $30^{a}$ semana de gestação, assim como as orientações recebidas pelas gestantes. Os autores descreveram que a assistência prestada foi insatisfatória nos dois modelos de atenção e que menos de $20 \%$, de ambos modelos de atenção, participaram de grupo de gestantes ou orientações em sala de espera.

\section{CONCLUSÃO}

O acompanhamento do estado nutricional materno durante o período gestacional constitui-se em um procedimento que deve incluir tanto 0 monitoramento do ganho de peso quanto do consumo alimentar. Portanto, a realização da orientação nutricional por profissional habilitado na assistência prénatal é fundamental para proporcionar a ingestão de uma alimentação equilibrada e adequada às necessidades fisiológicas da gestante.

Os resultados referentes aos desfechos gestacionais do presente estudo permitiram concluir que a assistência pré-natal foi efetiva para a prevenção de intercorrências gestacionais. Entretanto, não foi efetiva para influenciar na decisão da via de parto, pois a cesariana foi privilegiada em detrimento da via de parto preconizada pela Rede Cegonha, o parto normal.

É necessário, portanto, reduzir a realização de cesáreas, incentivando o atendimento humanizado na assistência pré-natal, parto e puerpério, estabelecendo 
critérios rigorosos para a indicação do parto cirúrgico e implementando o partograma, recurso recomendado pelo Ministério da Saúde.

\section{REFERÊNCIAS}

AMORIM, A. R.; ROSSNER, S.; NEOVIUS, M.; LOURENÇO, P. M.; LINNE, Y. Does excess pregnancy weight gain consitute a major risk for increasing long term BMI? Obesity. v. 15, n. 5, p. 1278-86, 2007.

ANDRETO, L. M.; SOUZA, A.I.; FIGUEIROA, J. N.; CABRAL FILHO, J. E. Fatores associados ao ganho ponderal excessivo em gestantes atendidas em um serviço público de pré-natal na cidade de Recife, Pernambuco, Brasil. Cad Saúde Pública v. 22, n. 11, p. 2401-09, 2006.

ANVERSA, E. T. R.; BASTOS, G. A. N.; NUNES, L. N.; PIZZOL, T.S. Qualidade do processo da assistência pré-natal: unidades básicas de saúde e unidades de Estratégia Saúde da Família em município no Sul do Brasil. Cad Saúde Pública. v. 28, n. 4, p. 789-800, 2012.

BAIÃO, M. R.; DESLANDES, S. F. Alimentação na gestação e puerpério. Rev Nutr. v. 19, n. 2, p. 245-253, 2006 .

BLOMBERG, M. Maternal and neonatal outcomes among obese women with weight gain below the new Institute of Medicine recommendations. Obstet Gynecol. v. 117, n. 5, p.1065-70, 2011.

BRASIL. Ministério da Saúde. Secretaria de Atenção à Saúde. Guia alimentar para a população brasileira : promovendo a alimentação saudável. Brasília: Ministério da Saúde, 2008.

BRASIL. Ministério da Saúde. Manual Prático para Implementação da Rede Cegonha. Brasília: Ministério da Saúde, 2011.

BUSS, C.; NUNES, M. A.; CAMEY, S.; MANZOLLI, P.; SOARES, R. M.; DREHMER, $M$. Dietary fibre intake of pregnant women attending general practices in southern Brazil-the ECCAGE Study. Public Health Nutr. v. 12, n. 9, p. 1392-8, 2009.

CORREIA, L.L.; SILVEIRA, D.M.I.; SILVA, A.C.; CAMPOS, J.S.; MACHADO, M.M.T.; ROCHA, H. A. L.; CUNHA, A. J. L. A.; LINDSAY, A. C. Prevalência e determinantes de obesidade e sobrepeso em mulheres em idade reprodutiva residentes na região semiárida do Brasil. Ciênc Saúde Coletiva. v. 16, n. 1, p. 13345, 2011.

DODD, J. M.; GRIVELL, R. M.; CROWTHER, C. A.; ROBINSON, J. S. Antenatal interventions for overweight or obese pregnant women: a systematic review of randomised trials. International J Obstet Gynaecol. v. 117, n. 11, p. 1316-26, 2010.

FALCONE, V.M.; MÄDER, C. V. N.; NASCIMENTO, C. L.; SANTOS, J. M. M.; NÓBREGA, F. J. Atuação multiprofissional e a saúde mental de gestantes. Rev Saúde Pública. v. 39, n. 4, p. 612-8, 2005. 
FAZIO, E. S.; NOMURA, R. M. Y.; DIAS, M. C. G.; ZUGAIB, M. Consumo dietético de gestantes e ganho ponderal materno após aconselhamento nutricional. Rev Bras Ginecol Obstet. v. 33, n. 2, p. 87-92, 2011.

GONÇALVES, C. V; MENDOZA-SASSI, R. A; CESAR, J. A.; BORTOLOMEDI, A. P. Índice de massa corporal e ganho de peso gestacional como fatores preditores de Complicações e do desfecho da gravidez. Rev Bras Ginecol Obstet. v. 34, n. 7, p. 304-9, 2012.

GRAVENA, A. A. F.; PAULA, M.G.; MARCON, S.S.; CARVALHO, M.D.B.; PELLOSO, S. M. Idade materna e fatores associados a resultados perinatais. Acta Paul Enferm. v. 26, n. 2, p. 130-5, 2013.

GUERRA, A. F. S.; HEYDE, M. E. D.; MULINARI, R.A. Impacto do estado nutricional no peso ao nascer de recém-nascidos de gestantes adolescentes. Rev Bras Ginecol Obstet. v. 29, n. 3, p. 126-33, 2007.

GUIMARÃES, A. F.; GALANTE, A. P. Anamnese Nutricional e Inquéritos Dietéticos. In: Avaliação Nutricional: Novas perspectivas. São Paulo: Roca, 2009. $42 p$.

GUIMARÃES, A. M. A. N.; BETTIOL, H.; SOUZA, L.; GURGEL, R. G.; ALMEIDA, M. L. D.; RIBEIRO, E. R. O.; GOLDANI, M. Z.; BARBIERI, M. A. Is adolescent pregnancy a risk factor for low birth weight? Rev Saúde Pública. v. 47, n. 1, p. 11-9, 2013.

HELENA, G.; SANTOS, N.; MARTINS, M. G.; SOUZA, M. S. Gravidez na adolescência e fatores associados com baixo peso ao nascer . Rev Bras Ginecol Obstet. v. 30, n. 5, p. 224-31, 2008.

INSTITUTE OF MEDICINE. National Academy of Sciences. Weight Gain During Pregnancy: Reexamining the Guidelines. Washington (DC): National Academy Press; 2009.

LUBCHENCO, L. O.; HANSMAN, C.; DRESSLER, M. Intrauterine growth as estimated from liveborn birthweity data at 24 to 42 weeks of gestation. Pediatrics. v. 45 , p. $937-944,1970$.

MARANO, D.; GAMA, S. G. N.; PEREIRA, A. P. E.; SOUZA JUNIOR, P. R. B. Adequação do ganho ponderal de gestantes em dois municípios do Estado do Rio de Janeiro (RJ), Brasil, 2008. Rev Bras Ginecol Obstet. v. 34, n. 8, p. 86-93, 2012.

MARTINS, A. P. B.; BENICIO, M. H. D. Influência do consumo alimentar na gestação sobre a retenção de peso pós-parto. Rev Saúde Pública. v. 45, n. 5, p. 870-77, 2011.

NASCIMENTO, R. M.; LEITE, A. J. M.; ALMEIDA, N. M. G.; SARAIVA, A. P. C.; SILVA, C. F. Determinantes da mortalidade neonatal: estudo caso-controle em Fortaleza, Ceará, Brasil. Cad Saúde Pública. v. 28, n. 3, p. 559-572, 2012. 
NELSON, S.M.; MATTHEWS, P.; POSTON, L. Maternal metabolism and obesity: modifiable determinants of pregnancy outcome. Hum Reprod. v. 16, n. 3, p. 255-75, 2010.

NOMURA, R. M. Y.; ALVES, E. A.; ZUGAIB, M. Complicações maternas associadas ao tipo de parto em hospital universitário. Rev Saúde Pública. v. 38, n. 1, p. 9-15, 2004.

NOMURA, R. M. Y.; PAIVA, L.V.; COSTA, V.N.; LIAO, A.W.; ZUGAIB, M. Influência do estado nutricional materno, ganho de peso e consumo energético sobre 0 crescimento fetal, em gestações de alto risco. Rev Bras Ginecol Obstet. v. 34, n. 3, p. 107-12, 2012.

PADILHA, P. C.; SAUDERS, C.; MACHADO, R.C.M.; SILVA, C.L.; BULL, A.S.; FONSECA, E.O.; ACCIOLY, E. Associação entre o estado nutricional prégestacional e a predição do risco de intercorrências gestacionais. Rev Bras Ginecol Obstet. v. 29, p. 511-518, 2007.

RASMUSSEN, K. M.; YAKTINE, A.L. Committee to Reexamine IOM Pregnancy Weight Guidelines, Food and Nutrition Board and Board on Children, Youth, and Families. Weight gain during pregnancy: reexamining the guidelines. Washington (DC): The National Academies Press; 2009.

RATTNER, D.; RABELLO NETO, D.L.; LANSKY, S.; VILELA, M. E.; BASTOS, M. H. As cesarianas no Brasil: situação no ano de 2010, tendências e perspectivas. In: Brasil. Ministério da Saúde. Secretaria de Vigilância em Saúde. Departamento de Análise de Situação de Saúde. Saúde Brasil 2011: uma análise da situação de saúde e a vigilância da saúde da mulher. Brasília: Ministério da Saúde, p. 371397.2012 .

REIS, Z. S. N; LAGE, E. M; AGUIAR, R. A. L. P; GASPAR, J. S; VITRAL, G. L. N; MACHADO, E. G. Associação entre risco gestacional $\square$ e tipo de parto com as repercussões maternas e neonatais. Rev Bras Ginecol Obstet. v. 36, n. 2, p. 6571, 2014.

SANTOS, G. H. N.; MARTINS, M. G.; SOUSA, M. S. Gravidez na adolescência e fatores associados com baixo peso ao nascer. Rev Bras Ginecol Obstet. v. 30, n. 5, p. 224-31, 2008.

SANTOS, M. M. A. S.; BAIÃO, M. R.; BARROS, D. C.; PINTO, A.A.; PEDROSA, P. $\mathrm{M}$; SAUDERS, C. Estado nutricional pré- gestacional, ganho de peso materno, condições da assistência pré-natal e desfechos perinatais adversos entre puérperas adolescentes. Rev Bras Epidemiol. v. 15, n. 1, p. 143-54, 2012.

SAUNDERS, C.; ACCIOLY, E.; COSTA, R. S. S.; LACERDA, E. M. A.; SANTOS, M. M. A. S. Gestante Adolescente. In: ACCIOLY, E.; SAUNDERS, C.; LACERDA, E. M. A. Nutrição em Obstetrícia e Pediatria. Rio de Janeiro: Cultura Médica; p.149$172,2009$. 
SEABRA, G.; PADILHA, P. C.; QUEIROZ, J. A.; SAUNDERS, C. Sobrepeso e obesidade pré-gestacionais: prevalência e desfechos associados à gestação. Rev Bras Ginecol Obstet. v. 33, n. 11, p. 348-53, 2011.

SOUZA, V.B.; ROECKER, S.; MARCON, S. S. Ações educativas durante a assistência pré-natal: percepção de gestantes atendidas na rede básica de MaringáPR. Revista Eletrônica de Enfermagem. v. 13, n. 2, p. 199-210, 2011.

SURITA, F. G. C.; SUAREZ, M. B. B.; SIANI, S.; SILVA, J. L. P. Fatores associados ao baixo peso ao nascimento entre adolescentes no Sudeste do Brasil. Rev Bras Ginecol Obstet. v. 33, n. 10, p. 286-291, 2011.

VIEIRA, S. M.; BOCK, L. F.; ZOCCHE, D. A.; PESSOTA, C. U. Percepção das puérperas sobre a assistência prestada pela equipe de saúde no pré-natal. Texto Contexto Enferm. v. 20, p. 255-62, 2011.

VITOLO, M. R.; BUENO, M. S. F.; GAMA, C. M. Impacto de um programa de orientação dietética sobre a velocidade de ganho de peso de gestantes atendidas em unidades de saúde. Rev Bras Ginecol Obstet. v. 33, n. 1, p. 13-19, 2011.

WORLD HEALTH ORGANIZATION (WHO). Appropriate technology for birth. Lancet. v. 2, n. 8452, p. 436-437, 1985.

YAZDANI, S.; YOSOFYAPASHA, Y.; NASAB, B. H.; MOJAVERI, M. H.; BOUZARI, $Z$. Effect of maternal body mass index on pregnancy outcome and newborn weight. BMC Research Notes. v. 5, n. 34, p. 1-4, 2012. 